\title{
Galactic sources at neutrino telescopes
}

\author{
Viviana Niro ${ }^{a, *}$ \\ ${ }^{a}$ Université de Paris, CNRS, Astroparticule et Cosmologie, F-75013 Paris, France \\ E-mail: viviana.niro@apc.in2p3.fr
}

The HAWC telescopes has recently revealed new spectra for gamma-ray sources in the Galactic plane. We will consider, in particular, the eHWC J1825-134 source and show the prospects to detect this source at the $\mathrm{KM} 3 \mathrm{NeT}$ detector. Moreover, we present a detailed study of the gamma-ray sources eHWC J1907+063 and eHWC J2019+368 and found that a detection at $3 \sigma$ or more at the IceCube detector should be within reach of the next decade. We also consider the source $2 \mathrm{HWC}$ J1857+027, which is coincident with the location of an IceCube neutrino excess. A detection at $3 \sigma$ of this source will depend on the specific value of the flux, on the extension and on the cut-off energy. Finally, we will show the prospects to detect the source RX J1713.7-3946 with $\mathrm{KM} 3 \mathrm{NeT}$ in a fully hadronic and in a lepto-hadronic scenario.

$37^{\text {th }}$ International Cosmic Ray Conference (ICRC 2021)

July 12th - 23rd, 2021

Online - Berlin, Germany

\footnotetext{
*Presenter
} 
Neutrino telescopes are able to provide important information on the production mechanisms of cosmic rays, since they can probe unambiguously the hadronic particle acceleration scenario. Indeed, if cosmic rays lose part of their energy in hadronic processes, then from the decay of charged pions, also high-energy neutrinos are expected. The problem of the origin of galactic cosmic rays and the gamma-ray emission is one of the most important problems in high energy astrophysics $[1,2]$. The characteristic gamma-ray feature of a souce able to accelerate cosmic rays up to PeV energies is an hard spectrum that extends until at least several tens of $\mathrm{TeV}$.

A multi-messenger search is mandatory for the correct identification of a PeVatron source, in particular a gamma-ray experiment with detection sensitivity up to about $100 \mathrm{TeV}$ is of fundamental importance. Moreover, gamma-ray data are necessary to make correct estimation of neutrino fluxes from point-sources.

Several galactic sources, in particular the one detected by the the Milagro Collaboration, have been studied in connection to the possible detection at the IceCube detector with through-going muons [3-7].

Recently, the High Altitude Water Cherenkov Observatory (HAWC) has reported new data on galactic sources in recent years, see e.g. [8-10]. In the 2HWC catalogue [8], 39 gamma-ray sources were identified, with an optimal sensitivity at about $7 \mathrm{TeV}$ energies. Subsequently, in the eHWC catalogue of Ref. [10], nine sources were observed above $56 \mathrm{TeV}$, all of which are likely Galactic in origin.

Among these sources, the eHWC J1825-134 source, located in the southern sky, has been detected with an hard spectrum that extends up to multi-TeV energies, thus it represents a possible PeVatron source. Moreover, this is the brightest source detected by HAWC in the multi-TeV domain. For this reason, it is one of the first source that should be searched for with a neutrino telescope in the northern hemisphere, like KM3NeT [11]. The sources eHWC J1907+063 and eHWC J2019+368 were identified with the eHWC catalogue as well [10], as two of the three gamma-ray sources, together with the eHWC J1825-134 source, that emits above $100 \mathrm{TeV}$. Six other sources, that emits above $56 \mathrm{TeV}$, were identified, among which some were previously detected also by Milagro. The source $2 \mathrm{HWC}$ J1857+027 does not belong to the eHWC catalogue. Note, however, that IceCube has reported a p-value of about $2 \%$ from this source [12].

\section{HAWC sources}

The source eHWC J1825-134, analysed in Ref. [10], is located in the southern sky, at adeclination of $-13.37^{\circ}$, and its spectrum can be described by a power law with exponential cut-off:

$$
\frac{d N_{\gamma}}{d E_{\gamma}}=\phi_{0}\left(\frac{E_{\gamma}}{10 \mathrm{TeV}}\right)^{-\alpha_{\gamma}} \exp \left(-\frac{E_{\gamma}}{E_{\text {cut }, \gamma}}\right)
$$

with $E_{c u t, \gamma}$ being the cut-off energy of the gamma-ray spectrum, $\alpha_{\gamma}$ the spectral index and $\phi_{0}$ the flux normalized, see the specific values reported in Ref. [10].

Instead, for two of the brighest sources in the eHWC catalogue, eHWC J1907+063 and eHWC J2019+368, it was found that a better fit to the gamma-ray spectrum is given by a log parabola, instead of a 
power law:

$$
\frac{d N_{\gamma}}{d E_{\gamma}}=\phi_{0}\left(\frac{E_{\gamma}}{10 \mathrm{TeV}}\right)^{-\alpha_{\gamma}-\beta \ln (E / 10 \mathrm{TeV})}
$$

with $\alpha_{\gamma}$ the spectral index and $\phi_{0}$ the flux normalization, see values reported in Ref. [10] for these two sources.

The emission reported in the $2 \mathrm{HWC}$ catalogue, instead, is parametrized by a power law:

$$
\frac{d N_{\gamma}}{d E_{\gamma}}=\phi_{0}\left(\frac{E_{\gamma}}{7 \mathrm{TeV}}\right)^{-\alpha_{\gamma}}
$$

where no cut-off is being reported. For the explicit values of the parameters, $\phi_{0}$ and $\alpha_{\gamma}$, for the hypothesis of point-source and for extended emission within a uniform disk, we refer to Ref. [8]. An error of about $50 \%$ on the flux normalization was reported and an error of $0.1^{\circ}$ on the tested radius.

The 2HWC J1908+063 source has been studied in the recent source analysis search done by the IceCube collaboration, and a p-value of about $1 \%$ from this source was reported, see Ref. [25].

The 2HWC J2019+367 source, instead, has been detected in the 2HWC catalogue within a radius of $0.7^{\circ}$. The source belongs to the Cygnus region, that is a star-forming region of about $5^{\circ}$ where five 2HWC sources can be found. A more complex picture for the 2HWC J2019+367 source might be present, i.e. neutrinos could be produced in association with supernova remnants and molecular clouds [13]. Considering the complexity of the Cygnus region, we have analysed for this source the extended emission from a radius of $0.7^{\circ}$, as reported in the $2 \mathrm{HWC}$ catalogue, as well as the parametrization reported in the eHWC catalogue [10], with an extension of $0.3^{\circ}$.

The source $2 \mathrm{HWC} \mathrm{J} 1857+027$ has been classified in the $2 \mathrm{HWC}$ catalogue and studied in connection with the IceCube data in Ref [12]. An excess of neutrinos was found from this region, resulting in a p-value of about $2 \%$. This source, however, does not belong to the eHWC catalogue, that contains the sources that emits above $56 \mathrm{TeV}$.

Finally, the source RX J1713-3946 is located in the southern sky with a declination of $-38.24^{\circ}$ and, for this reason, we investigated the possible detection of the source with the KM3NeT detector, considering a fully hadronic or a lepto-hadronic scenario.

\section{The neutrino flux at neutrino telescopes}

The event rate at KM3NeT can be described by the expression reported in Ref. [14]:

$$
N_{\mathrm{ev}}=\epsilon_{\theta} \epsilon_{v} t \int_{E_{v}^{\mathrm{th}}} d E_{v} \frac{d N_{v}\left(E_{v}\right)}{d E_{v}} \times A_{v}^{\mathrm{eff}}\left(E_{v}, \cos \theta_{Z}\right),
$$

where a sum over neutrino and antineutrino contributions is implicit. The parameter $\epsilon_{v}$ is the visibility of the source $\left(\epsilon_{v}=0.57\right.$ for the source eHWC J1825-134 and $\epsilon_{v}=0.7$ for the sources RX J1713-3946), while $\epsilon_{\theta}=0.72$ takes into account a reduction factor due to the fact that only a fraction of the signal will be detected if the source morphology is assumed to be a Gaussian of standard deviation $\sigma_{\text {ext }}$.

For the effective area, we considered the Letter of Intent of the KM3NeT collaboration which 

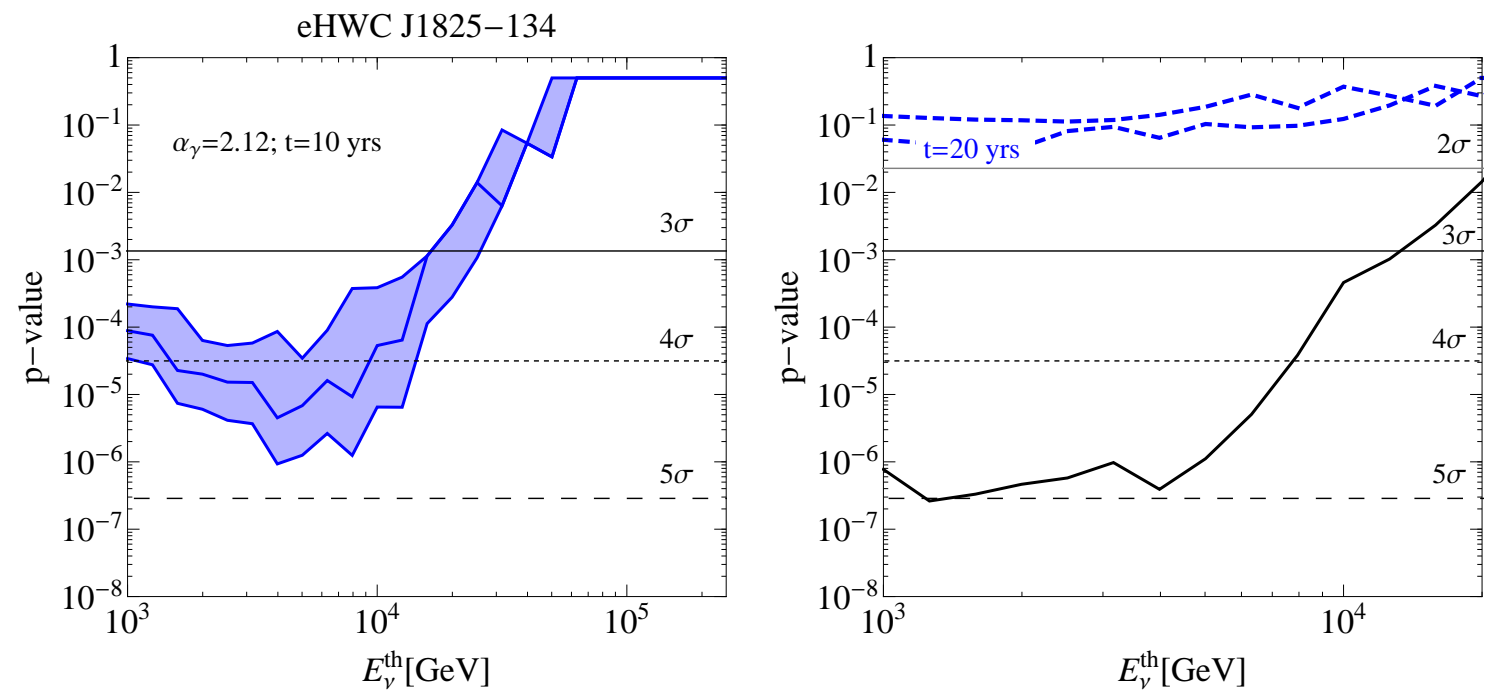

Figure 1: Left panel: p-value for the best-fit value of $\alpha_{\gamma}$ and values of $E_{c u t, \gamma}$ within the statistica errors, for 10 years of running of the KM3NeT detector. The normalization is fixed to the best-fit value and the extension of the source is fixed to $0.53^{\circ}$. Right panel: Statistical significance expected at the KM3NeT detector for the RX J1713.7-3946 source in a fully hadronic scenario and in a lepto-hadronic model.

Figures taken from [11, 23].

contains the expected performance of the KM3NeT/ARCA detector [15]. For the IceCube detector, instead, we have used the effective area reported in Ref. [16], where different bands in the zenith angle $\theta_{z}$ have been considered: $-1.00 \leq \cos \theta_{z} \leq-0.75,-0.75 \leq \cos \theta_{z} \leq-0.50$, $-0.50 \leq \cos \theta_{z} \leq-0.25$ and $-0.25 \leq \cos \theta_{z} \leq 0.08$.

We have considered $\sigma_{\text {res }} \sim 0.1^{\circ}$ as the angular resolution of KM3NeT/ARCA [15] detector, and $\sigma_{\text {res }} \sim 0.4^{\circ}[17]$ for the IceCube detector.

The number of neutrino events $\frac{d N_{v}\left(E_{v}\right)}{d E_{v}}$ has been calculated starting from the gamma-ray spectrum and considering the expressions given in Ref. [14, 18]. The expected atmospheric muon neutrinos [19-21] is integrated over an opening angle equal to $\Omega=\pi \sigma_{\text {eff }}^{2}$, where this effective angle contains information on the resolution of the detector and on the extension of the source.

For the eHWC J1907+063 source, we have also estimated the neutrino event rate at KM3NeT considering a more extended region of $1.5^{\circ}$ radius, as derived using also Fermi-LAT data, and we refer to [11] for more details on the topic.

\section{Statistical significance}

We have estimated the statistical significance as reported in Ref. [22] and as described in Refs. [6,7].

We report in the left panel of Fig. 1 the results for the p-value as a function of the neutrino energy threshold for 10 years of running time of the KM3NeT detector. For an energy threshold of the order of $E_{v}^{\text {thr }} \lesssim 10 \mathrm{TeV}$ a minimum in the p-value is present. Moreover, independently of the statistical error of the cut-off energy, the significance is well above $3 \sigma$ as long as the energy threshold is less than about $10 \mathrm{TeV}$. In the right panel of Fig. 1, we show the p-value as a function of the 
neutrino energy threshold for the source RX J1713.7-3946 [23]. If the gamma-ray emission is fully hadronic, a $5 \sigma$ discovery can be reached in 10 years of running of the KM3NeT detector for an energy threshold smaller then about $5 \mathrm{TeV}$. Instead, considering 10 years of running of the KM3NeT detector, it is difficult to resolve with a neutrino signal the mixed scenario of leptonic and hadronic emission. However, with an higher running time, of about 20 years, a p-value of the order of few percent could be reached.

We report in Fig. 2 the results for the p-value as a function of the neutrino energy threshold for the eHWC J1907+063 source and for 20 years of running time of the IceCube detector, see [24] for the case of 10 years running time. A significance of the order of $3 \sigma$ is reached for an energy threshold of about $1 \mathrm{TeV}$. Note that the IceCube detector currently has reported a p-value of about $1 \%$ from this source [25]. This source, previously identified by the Milagro experiment as MGRO J1908+06, was already considered as one of the most promising source to be detected by IceCube [3-7].

In the right panel of Fig. 2 we show the results for the p-value as a function of the energy threshold for 20 years of running time of the IceCube detector for the eHWC J2019+368 parametrization and extension. A detection with a p-value of $3 \sigma$ or more in 20 years running time is feasable. If $50 \%$ of the emission is leptonic, then the $3 \sigma$ will not be reached in 20 years of running time.

In Fig. 3, we report the result for the 2HWC J2019+367 parametrization and extension, for which almost $4 \sigma$ could be reached in 20 years, for an energy threshold of about $10 \mathrm{TeV}$ and for a cut-off energy of about $300 \mathrm{TeV}$.

Finally, we report in the right panel of Fig. 3 the results for the p-value as a function of the energy

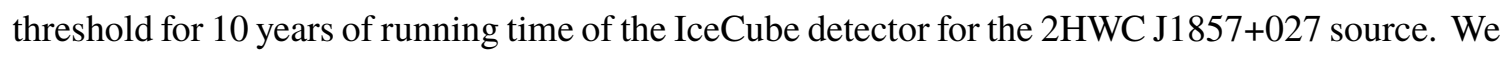
have considered the systematic error in the normalization of the flux and we have varied the cut-off energy, considering 50,100, $300 \mathrm{TeV}$. Moreover, we have considered the circular bin reported in the $2 \mathrm{HWC}$ catalogue, $0.9^{\circ}$, as well as a gaussian morphology with extension of $0.2^{\circ}$. The IceCube detector has reported a p-value of about $2 \%$ from this region. Within our statistical method, this could be possible only considering the systematic error on the normalization of the flux. Moreover, a better agreement is found considering a gaussian morphology with extension of about $0.2^{\circ}$ for the source and an energy cut-off greater than $100 \mathrm{TeV}$. Since this source has not been detected in the eHWC catalogue, additional data to clarify the situation are needed.

\section{Conclusions}

Using updated information on the gamma-ray spectrum provided by the HAWC collaboration, we have calculated the statistical significance expected at the $\mathrm{KM} 3 \mathrm{NeT}$ and the IceCube detectors for sources that are among the brighest in the eHWC and 2HWC catalogue.

For KM3NeT we have considered the sources eHWC J1825-134 and in addition RX J1713.7-3946. For IceCube, we have considered two of the brightest sources in the eHWC catalogue, eHWC $\mathrm{J} 1907+063$ and eHWC J2019+368, as well as the extended region 2HWC J2019+367. Moreover, since an excess in neutrinos is present from $2 \mathrm{HWC} \mathrm{J} 1857+027$, we have also studied the neutrino emission and the statistical significance for this source.

For the sources eHWC J1825-134 and RX J1713.7-3946 we found that the p-value can reach $4 \sigma$ and $5 \sigma$ respectively, for an energy threshold lower than $10 \mathrm{TeV}$. Moreover, we found that the significance can reach $3 \sigma$ or more in the next decade, for the sources eHWC J1825-134, eHWC J1907+063 and 

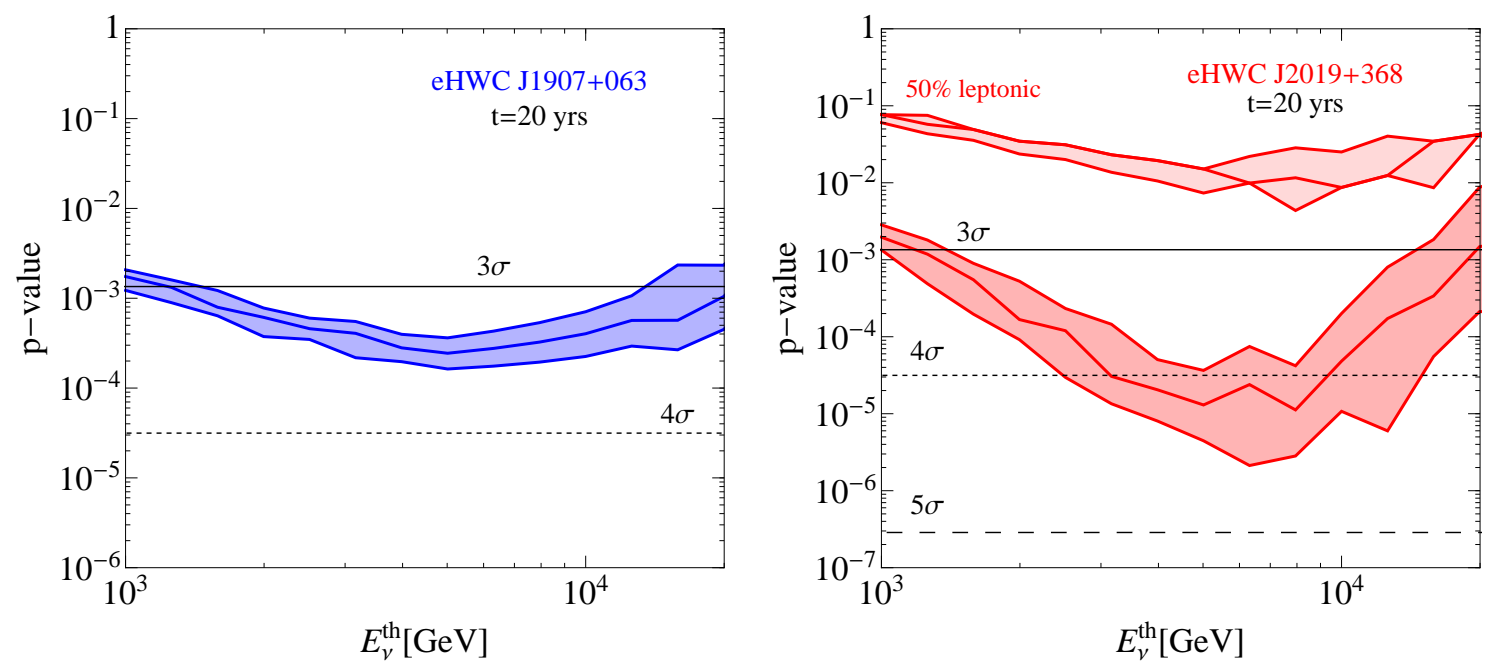

Figure 2: Left panel: Statistical significance expected at the IceCube detector for the source eHWC J1907+063 after 20 years running time. Right panel: Same as the left panel but for the source eHWC J2019+368.

Figures taken from [24].
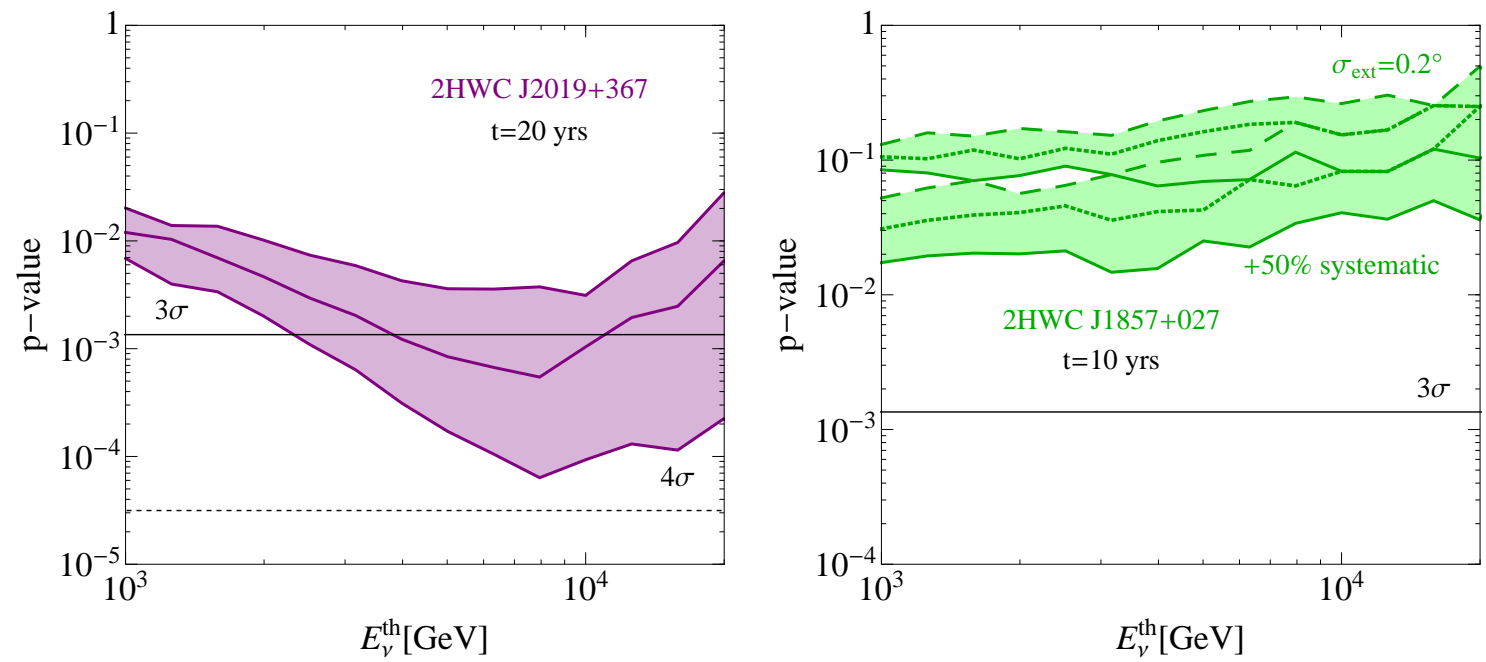

Figure 3: Left panel: Statistical significance expected at the IceCube detector for the source 2 HWC J2019+367 after 20 years running time. Right panel: Same as the left panel but for the source

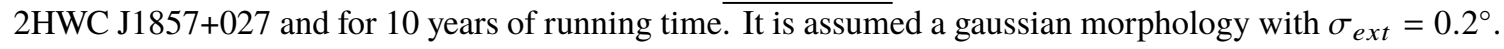
Figures taken from [24].

eHWC J2019+368. Considering the 2HWC J2019+367 region, instead, a detection at about $3 \sigma$ or more is expected in 20 years of running time, but for a neutrino energy threshold of about $10 \mathrm{TeV}$. For the source $2 \mathrm{HWC} \mathrm{J} 1857+027$ we have explicitly shown the dependence on the systematic error of the normalization and on the cut-off energy for the p-value. Assuming a gaussian morphology with an extension of $0.2^{\circ}$ or smaller, an high value of the normalization (within the $+50 \%$ error), 
and a cut-off energy of $100 \mathrm{TeV}$ or higher, we find a p-value of a few percent in 10 years and approaching $3 \sigma$ in the next decade. Future gamma-ray and neutrino data are needed to verify the gamma-ray emission above $56 \mathrm{TeV}$ of this source and the excess in neutrino data.

\section{Acknowledgments}

This project has received funding from the European Union's Horizon 2020 research and innovation programme under the Marie Skłodowska-Curie grant agreement No. 843418 (nuHEDGE).

\section{References}

[1] S. Gabici, C. Evoli, D. Gaggero, P. Lipari, P. Mertsch, E. Orlando, A. Strong, A. Vittino, Int. J. Mod. Phys. D 28 (15) (2019) 1930022. arXiv: 1903.11584.

[2] M. Kachelriess, D. Semikoz, Prog. Part. Nucl. Phys. 109 (2019) 103710. arXiv: 1904.08160.

[3] F. Halzen, A. Kappes, A. O'Murchadha, Prospects for identifying the sources of the Galactic cosmic rays with IceCube, Phys.Rev. D78 (2008) 063004. arXiv: 0803.0314.

[4] A. Kappes, F. Halzen, A. O. Murchadha, Prospects of identifying the sources of the galactic cosmic rays with IceCube, Nucl.Instrum.Meth. A602 (2009) 117-119.

[5] M. Gonzalez-Garcia, F. Halzen, S. Mohapatra, Identifying Galactic PeVatrons with Neutrinos, Astropart.Phys. 31 (2009) 437-444. arXiv: 0902 . 1176.

[6] M. C. Gonzalez-Garcia, F. Halzen, V. Niro, Reevaluation of the Prospect of Observing Neutrinos from Galactic Sources in the Light of Recent Results in Gamma Ray and Neutrino Astronomy, Astropart. Phys. 57-58 (2014) 39-48. arXiv: 1310 . 7194.

[7] F. Halzen, A. Kheirandish, V. Niro, Prospects for Detecting Galactic Sources of Cosmic Neutrinos with IceCube: An Update, Astropart. Phys. 86 (2017) 46-56. arXiv: 1609 . 03072.

[8] A. U. Abeysekara, et al., The 2HWC HAWC Observatory Gamma Ray Catalog, Astrophys. J. 843 (1) (2017) 40. arXiv: 1702 . 02992.

[9] K. A. Malone, A Survey of the Highest-Energy Astrophysical Sources with the HAWC Observatory, Ph.D. thesis, Penn State U. (2018).

[10] A. Abeysekara, et al., Multiple Galactic Sources with Emission Above $56 \mathrm{TeV}$ Detected by HAWC, Phys. Rev. Lett. 124 (2) (2020) 021102. arXiv: 1909. 08609.

[11] V. Niro, A. Neronov, L. Fusco, S. Gabici and D. Semikoz, Neutrinos from the gamma-ray source eHWC J1825-134: Predictions for Km3 detectors, Phys. Rev. D 104 (2021) no.2, 023017. arXiv: 1910.09065.

[12] A. Kheirandish, J. Wood, IceCube Search for Galactic Neutrino Sources based on HAWC Observations of the Galactic Plane, in: 36th International Cosmic Ray Conference (ICRC 2019) Madison, Wisconsin, USA, July 24-August 1, 2019, 2019. arXiv: 1908.08546. 
[13] S. Gabici, F. A. Aharonian, Searching for galactic cosmic ray pevatrons with multi-TeV gamma rays and neutrinos, Astrophys. J. Lett. 665 (2007) L131. arXiv: 0705 . 3011.

[14] A. Kappes, J. Hinton, C. Stegmann, F. A. Aharonian, Astrophys.J. 656 (2007) 870-896. arXiv: astro-ph/0607286.

[15] S. Adrian-Martinez, et al., Letter of intent for KM3NeT 2.0, J. Phys. G43 (8) (2016) 084001. arXiv: 1601.07459.

[16] J. Stettner, Measurement of the Diffuse Astrophysical Muon-Neutrino Spectrum with Ten Years of IceCube Data, PoS ICRC2019 (2020) 1017. arXiv: 1908. 09551.

[17] M. G. Aartsen, et al., All-sky Search for Time-integrated Neutrino Emission from Astrophysical Sources with $7 \mathrm{yr}$ of IceCube Data, Astrophys. J. 835 (2017) no.2, 151. arXiv: 1609.04981.

[18] F. L. Villante, F. Vissani, How precisely neutrino emission from supernova remnants can be constrained by gamma ray observations?, Phys. Rev. D78 (2008) 103007. arXiv: 0807.4151.

[19] M. Honda, T. Kajita, K. Kasahara, S. Midorikawa, Improvement of low energy atmospheric neutrino flux calculation using the JAM nuclear interaction model, Phys.Rev. D83 (2011) 123001. arXiv: 1102.2688.

[20] L. Volkova, Energy Spectra and Angular Distributions of Atmospheric Neutrinos, Sov.J.Nucl.Phys. 31 (1980) 784-790.

[21] P. Gondolo, G. Ingelman, M. Thunman, Charm production and high-energy atmospheric muon and neutrino fluxes, Astropart.Phys. 5 (1996) 309-332. arXiv: hep-ph/9505417.

[22] Procedure for the LHC Higgs boson search combination in summer 2011, ATL-PHYS-PUB2011-011, CMS-NOTE-2011-005.

[23] P. Cristofari, V. Niro and S. Gabici, Gamma rays and neutrinos from RX J1713.7-3946 in a lepto-hadronic scenario, arXiv:2105.12494.

[24] V. Niro, Detecting the brightest HAWC sources with IceCube in the upcoming years, Phys. Rev. D 103, no.10, 103020 (2021). arXiv: 2012 . 02599.

[25] M. Aartsen, et al., Search for steady point-like sources in the astrophysical muon neutrino flux with 8 years of IceCube data, Eur. Phys. J. C 79 (3) (2019) 234. arXiv: 1811.07979. 